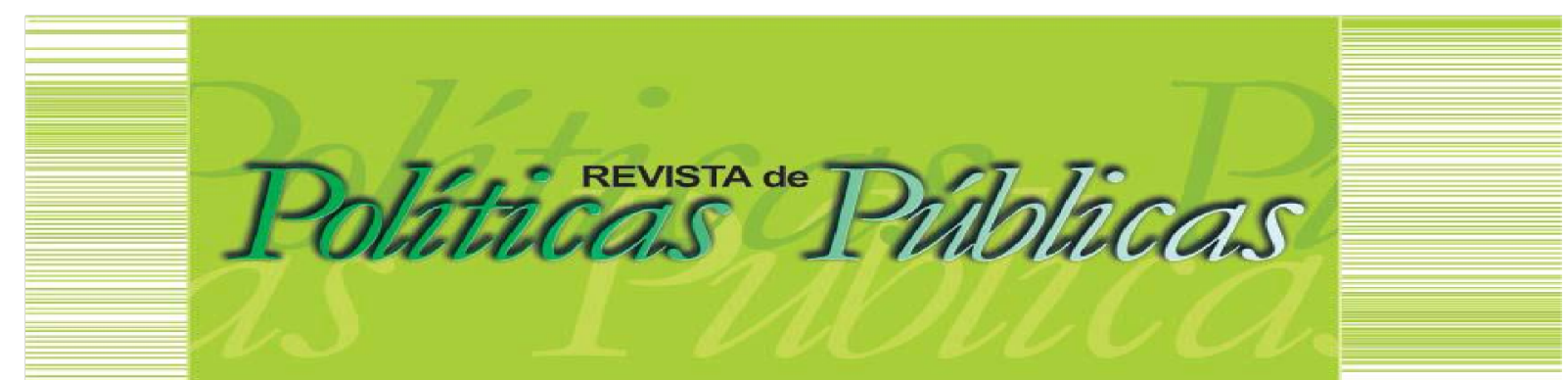

\title{
INVESTIMENTO EM SANEAMENTO BÁSICO E A MELHORIA DAS CONDIÇÕES DE SAÚDE DAS CAPITAIS ESTADUAIS BRASILEIRAS
}

\author{
Gleice Carvalho de Lima Moreno1 \\ Douglas Heinz ${ }^{2}$ \\ Nelson Hein ${ }^{3}$
}

\begin{abstract}
Resumo
A investigação dos efeitos do saneamento básico sobre a saúde tem recebido crescente atenção acadêmica, mas ainda são necessárias evidências empíricas que destaquem os resultados dessa relação. Visando corroborar com esta abordagem, este estudo avalia o impacto dos investimentos e ações de saneamento básico na melhoria das condições de saúde das 27 capitais das unidades da federação do Brasil. Resulta, portanto, de umapesquisadescritiva, de abordagem quantitativa, utilizando técnica de regressão linear múltipla com análise de dados em painelaplicada aos dados secundários coletados no Sistema Nacional de Informações Sobre Saneamento (SNIS) e no índice FIRJAN de saúde. Os resultados obtidos indicam que o percentual da população atendida por rede de esgotamento sanitário é o principal item de saneamento básico que impacta na variação das condições de saúde das capitaispelo seu baixo desempenho.
\end{abstract}

Palavras-chave:Saúde pública. Saneamento básico. Capitais Brasileiras.

\section{INVESTMENT IN BASIC SANITATION AND THE IMPROVEMENT OF HEALTH CONDITIONS IN BRAZILIAN STATE CAPITALS}

\begin{abstract}
The investigation of the effects of basic sanitation on health has received increasing academic attention, but empirical evidence is still needed to highlight the results of this relationship. Aiming to corroborate this approach, this study assesses the impact of investments and basic sanitation actions in improving the health conditions of the 27 capitals of the Brazilian federation units. It is a descriptive research, with a quantitative approach, using multiple linear regression technique with panel data analysis applied to secondary data collected in the National Sanitation Information System (SNIS) and in the FIRJAN health index. The results obtained indicate that the percentage of the population served by the sewage system is the main item of basic sanitation that impacts on the variation of the health conditions of the capitals due to its low performance. Keywords: Public Health. Basic sanitation. Brazilian Capitals.
\end{abstract}

Artigo recebido em: 05/07/2021 Aprovado em: 20/11/2021 DOl: http://dx.doi.org/10.18764/2178-2865.v25n2p 908-920

\footnotetext{
${ }^{1}$ Doutoranda em Ciências Contábeis e Administração pela Universidade Regional de Blumenau (FURB). Mestre em Ciências Contábeis pela Universidade Federal de Pernambuco (UFPE). Docente do Departamento de Ciências Contábeis da Universidade Federal de Rondônia (UNIR) em Porto Velho.E-mail: gleicemoreno@unir.br

2 Graduado em Administração de Empresas. Doutorando em Ciências Contábeis e Administração pela Universidade Regional de Blumenau (FURB). Docente do Departamento de Administração da UNIDAVI. E-mail: dh@unidavi.edu.br 3 Doutor em Engenharia de Produção pela Universidade Federal de Santa Catarina. Professor titular da Fundação Universidade Regional de Blumenau, Brasil. Docente do Departamento de Matemática e da Pós-Graduação em Ciências Contábeis e Administração da Universidade Regional de Blumenau. E-mail: hein@furb.br
} 


\section{INTRODUÇÃO}

O serviço público de saneamento básico, por suas características, pode ser considerado como essencial para a saúde, no sentido de que sua ausência implica uma série de consequências que podem prejudicar o bem-estar, a qualidade e a expectativa de vida de cada cidadão (LAHOZ; DUARTE, 2015). Apesar disso, a área de saneamento ainda carece de aproximação com as perspectivas da saúde pública. $O$ aprofundamento da compreensão dessa relação pode ter importante efeito sobre as práticas de ambas as áreas (HELLER, 1998).

Um aumento nos investimentos em saneamento pode ser considerado como parte de uma estratégia de desenvolvimento econômico e social (MADEIRA, 2010), mas mesmo com esse reconhecimento, e a despeito das carências observadas, os recursos financeiros disponíveis para 0 setor são escassos no Brasil (TEIXEIRA; GUILHERMINO, 2006). Além disso, o crescimento desordenado das cidades, aliado ao emprego inadequado das operações com o saneamento básico, têm gerado a piora no quadro epidemiológico e causado sérios danos ao meio ambiente (MACIEL et al., 2015).

O que pode se perceber é que em países subdesenvolvidos os recursos são escassos para 0 atendimento das políticas públicas, dentre elas, a política pública de saneamento básico que acaba sendo um serviço precário, favorecendo o agravamento de doenças e deixando a saúde pública em más condições (FERREIRA et al., 2016). No Brasil, a Lei Nacional do Saneamento básico já prevê a priorização dos investimentos em política pública específica abrangendo os aspectos social, de saúde e ambiental (BORJA, 2014). Mesmo assim, as regras que definem direitos e deveres não garantem o acesso universal e de qualidade a todos os brasileiros.

Nesse campo, uma das lacunas que merece mais investigações é a análise dos efeitos específicos de intervenções de saneamento sobre distintos agravos à saúde (HELLER, 1998). Estudos existentes costumam se concentrar em identificar os impactos do saneamento em um aspecto específico da saúde, como a mortalidade infantil (BARBIERI et al. 2015) ou óbitos causados por doenças relacionadas à falta de saneamento (TEIXEIRA et al., 2014). Percebe-se que tais pesquisas utilizam apenas casos extremos (mortes) para avaliar as condições de saúde, deixando de considerar outros efeitos dessa relação. Deste modo, fatores como urbanização, infraestrutura básica, desenvolvimento industrial, conservação ambiental (qualidade da água e controle da poluição), educação sanitária e serviços de saúde pública conduzem às ações de saneamento básico e representam importantes pontos para discussão e desenvolvimento de pesquisas, como de Soares et al. (2002), Ferreira et al.(2016), Melo et al. (2017) e Borja (2014), envolvendo ainda os aspectos econômico, social, político, financeiro e institucional de maior complexidade. 
A construção teórica mais fundamentada sobre as políticas públicas de saneamento e para a avaliação de experiências empíricas, com consistência metodológica, pode contribuir para uma maior qualificação da formulação na área, para maior qualidade das reflexões e dos debates que envolvem esse campo, o que, no longo prazo, pode resultar em políticas públicas mais orientadas para os interesses da população, aumentando a efetividade e a eficiência das iniciativas. (HELLER; CASTRO, 2007)

Assim, o presente estudo avalia o impacto dos investimentos e ações de saneamento básico na melhoria das condições de saúde das 27 capitais das unidades da federação do Brasil, utilizando como variável de análise das condições de saúde o indicador FIRJAN de saúde dos municípios, que é composto por elementos adicionais, como internações sensíveis à atenção básica (FIRJAN, 2018).

O conteúdo está organizado da seguinte forma: após esta introdução há uma revisão da literatura científica sobre a relação entre o saneamento básico e a saúde, seguida da apresentação dos procedimentos metodológicos utilizados, da análise dos dados e discussão dos resultados e das considerações finais.

\section{A RELAÇÃO ENTRE SANEAMENTO BÁSICO E SAÚDE}

Os dados históricos expõem que o saneamento básico está presente há muito na história da humanidade, assumindo conteúdos diferenciados, em virtude de fatores culturais que refletem a relação do homem com a natureza. A origem da palavra saneamento vem do latim sanus, com vários significados. Dentre eles, temos como exemplo a expressão tornar são, que representa de forma precisa a relação com a saúde (MORAES; BORJA, 2014). Além disso, essa expressão reproduz um conjunto de ações com o propósito de prevenir doenças e melhorar a qualidade de vida da população (MACIEL; FELIPE; LIMA, 2015), impedindo que doenças de veiculação sanitária ou hídrica sejam constantes no meio social (MOURA; TROLEl; SILVA, 2016).

É uma política pública essencial para a sociedade por ter como ações o suprimento de água potável, o tratamento da rede de esgoto, a coleta e o tratamento dos resíduos sólidos e orgânicos (lixo) em aterros sanitários e a realização do manejo de águas pluviais. Essas ações estão diretamente associadas à promoção da saúde pública e, consecutivamente, à segurança e duração da vida, além da conservação ambiental (FERREIRA et al., 2016). Entretanto, mesmo com ações promissoras propostas pelo governo brasileiro até 2015 , o abastecimento de água supria cerca de $83,3 \%$ da população brasileira e apenas $50,3 \%$ dos brasileiros tinham acesso ao tratamento de esgoto (SNIS, 2015). Como se vê, a garantia aos direitos básicos, como o acesso à água e ao destino seguro dos 
dejetos e resíduos sólidos, é interrompida à medida que surgem outros serviços públicos considerados, também, essenciais, não havendo recursos suficientes para atender as diversas necessidades da sociedade (BORJA, 2014) pela falta de um bom planejamento.

A relação do saneamento com a saúde é percebida quando notamos que as ações pertinentes a essa política pública tendem a reparar a transmissão das doenças provenientes de categorias ambientais ou da falta de infraestrutura adequada (SOARES; BERNARDES; CORDEIRO NETTO, 2002). Outrossim, as mudanças climáticas agregadas à ausência de um bom saneamento podem agravar a incidência de doenças infecciosas, acarretar perdas na agricultura de subsistência, gerando a desnutrição e, ainda, mais problemas para as comunidades locais (MELO et al., 2017). Ademais, o uso de soluções técnicas universais relacionadas ao saneamento básico, para impedir 0 alastramento de doenças de origem sanitária, desconsiderando as particularidades locais, pode privilegiar alguns grupos em detrimento de outros (MENDES; BARCELLOS, 2018).

De certa forma, é notável que o Brasil ainda não superou alguns traços coloniais, concentrando o poder nas mãos de poucos e com isso reproduzindo reflexos para as populações mais carentes, que ficam expostas a uma série de infortúnios socioambientais (MARICATO apud DIAS et al., 2017). É o caso das populações rurais como as indígenas, os assentados, os quilombolas, que são excluídos das tecnologias de saneamento convencionais consideradas caras, deixando esses grupos com problemas de saúde em suas comunidades (MOTA; SOUSA; SILVA, 2015) à mercê da própria sorte e de um futuro incerto. Decorrente disso, é perceptível que as ofertas dos serviços pelo poder público são comuns em grandes aglomerações, tendo em vista a redução dos custos à medida que aumenta a população, tornando pública a motivação do emprego de recursos mais pelo retorno econômico do que pelo retorno social (SAIANI; TONETO JR., 2010). Esse fenômeno é percebido em outros estudos que exploram regiões menores, apresentando diversas vulnerabilidades na saúde pública resultantes da falta de saneamento básico.

Nesse sentido, o saneamento básico tem forte relação com a saúde quando nos deparamos com diversas pesquisas que confirmam os efeitos gerados pela má aplicação de recursos ou pela forma inadequada de conduzir as ações de saneamento em prol da sociedade, tais como Silva et al. (2017), Soares; Bernardes; Cordeiro Netto (2002), Dias; Raiol; Nonato(2017); Teixeira; Guilhermino (2006); Lima; Santos; Medeiros (2017); Lahos; Duarte (2015); Heller (1998); Barcellos et al. (2009); Rosa; Becerra; Lunkes (2016); entre outros. Partindo desse pressuposto, constatamos que as doenças mais comuns procedentes da implantação de um mau saneamento básico ou pela falta dele são: malária, dengue, febre amarela, leptospirose, cólera, esquistossomose, febre tifoide, diarreia, hepatites, desinteria bacilar, verminoses, doença de Chagas, conjuntivite, doenças de pele, entre 
outras, que estão presentes em todas as regiões brasileiras aliadas às mudanças ambientais e climáticas.

Em síntese, o saneamento básico, se não planejado de forma eficaz para atender as comunidades, pode acarretar iminentes riscos de saúde, principalmente para os grupos desprovidos de condições mínimas de existência. As mudanças climáticas e fatores associados, como a globalização, as transformações ambientais e a precarização de sistemas de governosão agravantes deste quadro (BARCELLOS et al., 2009). Sendo assim, enquanto não houver a universalização do saneamento, como um direito fundamental para a sociedade, o acesso à saúde não se concretizará de forma absoluta, exigindo mais esforços dos sistemas de governo para solucionar tal problema (LAHOS; DUARTE, 2015).

\section{METODOLOGIA}

A pesquisa é do tipo descritiva, que visa a identificação, registro e análise das características, fatores ou variáveis que se relacionam com o fenômeno em processo. Descrever significa dizer como se comporta um sistema em análise (JUNG, 2004).

Adotamos a abordagem quantitativa, que testa teorias objetivas examinando relações entre variáveis de forma numérica ou quantitativa. O corte aplicado foi longitudinal, com dados coletados no decorrer do tempo (CRESWELL, 2010).

A amostra da pesquisa adotada por conveniência (CRESWELL, 2010) é composta pelas 27 capitais das Unidades de Federação e do Distrito Federal do Brasil. Essa amostra foi escolhida considerando-se que as capitais possuem maior volume de dados disponíveis para análise, além de concentraram significativa parcela da população residente em cada unidade da federação, tornando a investigação conduzida relevante do ponto de vista de sua abrangência.

Foram coletados dados secundários entre os anos de 2005 a 2016 no sistema SNIS (Sistema Nacional de Informações sobre Saneamento) e no índice FIRJAN (Federação das Indústrias do Rio de Janeiro) de Saúde dos municípios. Os dados obtidos no SNIS buscam refletir políticas públicas de saneamento aplicadas nas capitais, tanto em relação a investimentos quanto à população beneficiada. Já o índice FIRJAN foi escolhido por ser uma boa representação das condições de saúde de cada município. Este índice é composto a partir de quatro informações: proporção de atendimento adequado no pré-natal, óbitos por causas mal definidas, óbitos infantis por causas evitáveis e internação sensível à atenção básica (FIRJAN, 2018).

O Quadro 1 detalha os dados levantados: 
Quadro 1 - Dados levantados no SNIS e Índice FIRJAN.

\begin{tabular}{|l|l|l|}
\hline Sigla & \multicolumn{1}{|c|}{ Dado } & Fonte \\
\hline POP_TOT & População total do Município no ano & SNIS \\
\hline AG001 & População do município atendida com abastecimento de água & SNIS \\
\hline ES001 & População do município atendida com esgotamento sanitário & SNIS \\
\hline FN006 & Arrecadação total do município & SNIS \\
\hline FN033 & Investimentos totais em saneamento básico realizados pelo prestador de serviço & SNIS \\
\hline FN048 & Investimentos totais em saneamento básico realizados pelo município & SNIS \\
\hline FN058 & Investimentos totais em saneamento básico realizados pelo estado & SNIS \\
\hline IFIRJANS & Índice Firjan de Saúde dos Municípios & FIRJAN \\
\hline
\end{tabular}

Fonte: Os autores.

Os dados permitiram a formulação das variáveis utilizadas no modelo de regressão linear elaborado, visando testar a hipótese do estudo. 0 quadro 2 destaca as variáveis e a forma como foram calculadas:

Quadro 2 - Variáveis utilizadas no modelo de regressão linear

\begin{tabular}{|l|l|l|}
\hline \multicolumn{1}{|c|}{ Tipo } & \multicolumn{1}{|c|}{ Nome } & \multicolumn{1}{|c|}{ Descrição } \\
\hline Dependente & IFIRJANS & Índice Firjan de Saúde dos Municípios \\
\hline Independente & \%INVEST & $\begin{array}{l}\text { Percentual de investimentos em saneamento básico em relação à } \\
\text { arrecadação total do município. } \\
\text { \%INVEST = (FN033 + FN0048 + FN058) / FN006 }\end{array}$ \\
\hline Independente & \%POPAG & $\begin{array}{l}\text { Percentual da população do município atendida com abastecimento de água. } \\
\text { \%POPAG = (AG001 / POP_TOT) }\end{array}$ \\
\hline Independente & \%POPES & $\begin{array}{l}\text { Percentual da população do município atendida com esgotamento sanitário. } \\
\text { \%POPES = (ES001 / POP_TOT) }\end{array}$ \\
\hline
\end{tabular}

Fonte: Os autores.

Para análise utilizamos a técnica de análise de dados em painel com auxílio do software IBM SPSS Statistics versão 22. Foi adotado o mesmo procedimento utilizado por Toigo, Wrubel e Hein (2014).

\section{ANÁLISE E DISCUSSÃO DOS RESULTADOS}

Os resultados estão divididos em duas partes, abrangendo as estatísticas descritivas e multivariadas dos dados. Ao final da seção discutimos as descobertas do estudo. 


\section{1 Estatística descritiva}

Como foram coletados dados das 27 capitais de estados e do Distrito Federal no intervalo de 12 anos, existem 324 observações para cada uma das variáveis. A tabela 1 apresenta as estatísticas descritivas das três variáveis independentes utilizadas.

Tabela 1 - Estatísticas descritivas para as variáveis independentes

\begin{tabular}{l|c|c|c|c|c}
\hline & $\mathrm{N}$ & Mínimo & Máximo & Média & Erro Desvio \\
\hline$\%$ Investimento & 324 &, 0000000000 & 3,689946160 &, 3170304464 &, 4357369291 \\
\hline$\%$ Água & 324 &, 1144868706 & 1,000000000 &, 8918693321 &, 1680569608 \\
\hline$\%$ Esgoto & 324 &, 0000000000 & 1,000000000 &, 4973484069 &, 2941578414 \\
\hline
\end{tabular}

Fonte: Dados da pesquisa.

As informações da Tabela 1 necessitam ser contextualizadas, em especial no que diz respeito ao percentual de investimento em saneamento básico, uma vez que o valor máximo é superior a 100\%, o que parece representar inconsistência nos dados. Para esclarecer este ponto é importante reforçar que esta variável é formada a partir dos somatórios dos investimentos realizados pelo município, pelo estado e pelo prestador de serviços, portanto os dados precisam ser entendidos como um montante, o que justifica o elevado percentual em alguns casos.

Já quanto a população atendida por serviços de água e saneamento,foi observada a elevada amplitude entre os valores mínimo e máximo destes dois serviços, o que evidencia diferenças significativas no seu alcance nas diferentes capitais ao longo do tempo. Paralelamente, a média da cobertura do abastecimento de água aproxima-se de $90 \%$, evidenciando que este é um serviço que apresenta elevada disponibilidade nas capitais brasileiras, ao contrário da cobertura da rede de esgotos, que não alcança sequer $50 \%$ da população, em média.

\section{2 Estatística multivariada}

Inicialmente obtivemos a matriz de correlação entre as variáveis dependentes e independentes. 
Tabela 2 - Correlação de Pearson entre as variáveis

\begin{tabular}{l|c|c|c|c}
\hline & Saúde_Firjan & $\%$ Investimento & \% Água & $\%$ Esgoto \\
\hline Saúde_Firjan & 1 &, 011 &, $582^{* *}$ &, $702^{* *}$ \\
\hline$\%$ Investimento & & 1 &,- 035 &,$- 158^{* *}$ \\
\hline$\%$ Água & & & 1 &, $607^{* *}$ \\
\hline$\%$ Esgoto & & & & 1 \\
\hline
\end{tabular}

Fonte: Dados da pesquisa

Foi observado que a variável independente (Saúde_Firjan) está correlacionada de forma positiva e significativa ao nível $p<0,01$ com as variáveis "\%Água" e "\%Esgoto". Este resultado indica que no período analisado o aumento nos percentuais de cobertura das redes de água e de esgoto tiveram impacto positivo no índice FIRJAN, o que em última instância significa uma influência positiva dos serviços sobre as condições de saúde da população, corroborando a principal premissa que fundamenta o desenvolvimento do estudo.

Além de ter sido possível constatar na Tabela 2, que a maior correlação entre as variáveis independentes foi de pouco mais de $60 \%$, este resultado é relevante para indicar que o modelo não apresentou multicolinearidade, que segundo Hair Jr. (2005) é a extensão em que uma variável pode ser explicada pelas outras variáveis da análise. Para Field (2004), uma das condições para que seja possivel tirar conclusões sobre uma população em um modelo de regressão é que as variáveis previsoras não apresentem correlações muito altas, indicando não existir multicolinearidade.

Com isso, foi utilizada a análise de regressão com dados em painel com as informações de todas as variáveis (TOIGO; WRUBEL; HEIN, 2014). A Tabela 3 apresenta o resumo do modelo.

Tabela 3 - Resumo do modelo

\begin{tabular}{l|l|l|l|l|l}
\hline Modelo & $\mathrm{R}$ & R quadrado & $\begin{array}{c}\text { R quadrado } \\
\text { ajustado }\end{array}$ & $\begin{array}{c}\text { Erro padrão da } \\
\text { estimativa }\end{array}$ & Durbin-Watson \\
\hline
\end{tabular}




\begin{tabular}{l|l|l|l|l|l}
\hline 1 &, $737^{\mathrm{a}}$ &, 543 &, 539 &, 0592932 & 1,594 \\
\hline
\end{tabular}

a. Preditores: (Constante), \% Esgoto, \% Investimento, \% Água

b. Variável Dependente: Saúde_Firjan

Fonte: Dados da pesquisa.

O coeficiente de determinação $\left(R^{2}\right)$ fornece uma medida de quanto a variabilidade da saída pode ser debitada dos previsores. Assim, conforme demonstra a tabela $3,0 \mathrm{R}^{2}=, 543$ indica que o modelo é eficaz para identificar a influência das variáveis em $54,3 \%$, ou seja, o modelo evidencia que as variáveis independentes são responsáveis por $54,3 \%$ da variação do índice FIRJAN. Já o $O R^{2}$ ajustado fornece uma noção de quão bem o modelo generaliza, sendo que, quanto mais próximo 0 valor do $R^{2}$, melhor é o poder explicativo do modelo. A diferença encontrada no modelo, de 0,004 é pequena, indicando que se o modelo fosse aplicado à população em vez da amostra, quase não existiriam diferenças.

Já o teste de Durbin-Watson verifica a presença de autocorrelação entre os resíduos. Quanto mais próximo de 2 for o valor encontrado, melhor (MAROCO, 2007). 0 resultado obtido foi de 1,594. Em relação ao resultado dos coeficientes da regressão, os valores são apresentados na Tabela 4.

Considerando que o valor encontrado da estatística VIF foi menor do que 10 , reforçamos a conclusão de que não há presença de multicolinearidade nos dados (FIELD, 2004), Ainda, todos os valores encontrados foram significativos no nível $p<0,05$.

Tabela 4 - Coeficientes

\begin{tabular}{l|c|c|c|c|c|c|c}
\hline & $\mathrm{B}$ & Erro & B padronizado & $\mathrm{t}$ & Sig. & Tolerância & VIF \\
\hline (Constante) &, 602 &, 019 & & 31,675 &, 000 & & \\
\hline$\%$ Investimento &, 022 &, 008 &, 110 & 2,879 &, 004 &, 969 & 1,032 \\
\hline$\%$ Água &, 123 &, 025 &, 236 & 4,962 &, 000 &, 628 & 1,591 \\
\hline$\%$ Esgoto &, 171 &, 014 &, 576 & 11,944 &, 000 &, 613 & 1,630 \\
\hline
\end{tabular}


a. Variável Dependente: Saúde_Firjan

Fonte: Dados da pesquisa

A partir dos resultados encontrados é possível construir a equação preditora do impacto das políticas de saneamento sobre a o índice FIRJAN de saúde:

$$
\text { Saúde_Firjan }=0,602+0,022 \% \text { Investimento }+0,123 \% \text { Água }+0,171 \% \text { Esgoto }
$$

Esses valores apontam que o fator de maior impacto para o aumento do índice FIRJAN, que representa as condições de saúde dos municípios, é o percentual da população atendida por rede de esgotos, seguido do percentual da população atendida por abastecimento de água e pelo investimento em saneamento básico.

Uma interpretação possível para essa descoberta é que indicadores de atendimento da população devem receber maior atenção por parte dos executores de políticas públicas de saneamento nas capitais estaduais, em detrimento de preocupações puramente econômicas, como o volume de recursos investidos. Observamos, pelos resultados encontrados no estudo, que o maior impacto nas condições de saúde da população se dá pela ampliação da cobertura da rede de esgoto, logo, melhorar este indicador deveria ser a preocupação primária em ações de saneamento básico.

Finalmente, esta constatação reforça a importância da eficaz aplicação dos limitados recursos disponíveis para o saneamento básico no país. Mesmo altos investimentos, se não resultarem na ampliação da cobertura do abastecimento de água e, principalmente, da rede de esgotos, podem trazer resultados de pouco impacto naquilo que seria a sua principal finalidade: a melhoria das condições de saúde da população.

\section{CONSIDERAÇÕES FINAIS}

O objetivo do estudo foi avaliar o impacto dos investimentos e ações de saneamento básico na melhoria das condições de saúde das 27 capitais das unidades da federação do Brasil. Para tanto, foram realizadas análises de estatística descritiva e multivariada de dados. Obteve-se um modelo de regressão por dados em painel capaz de explicar $54,3 \%$ da variabilidade das condições de saúde da população a partir das variáveis preditoras, percentual de investimentos em saneamento básico em relação à arrecadação total do município, percentual da população do município atendida com abastecimento de água e percentual da população do município atendida com esgotamento sanitário.

Como resultado, identificamos que o fator de maior impacto para o aumento do índice FIRJAN, que representa as condições de saúde dos municípios, é o percentual da população atendida 
por rede de esgotos. Tal descoberta aponta para a necessidade dos responsáveis por políticas públicas de saneamento priorizarem a melhoria deste indicador no planejamento de ações na área. Considerando ainda que os dados demonstram um nível médio de atendimento da população por esgotamento sanitário nas capitais federais inferior a 50\%, observamos que 0 aspecto do saneamento básico de maior importância para a saúde é justamente aquele mais negligenciado e de menor disponibilidade para a população. Corrigir tal distorção deveria ser prioridade para a administração municipal nas capitais estaduais brasileiras.

Algumas limitações estão presentes neste estudo. Primeiro, como foram utilizados apenas dados das capitais estaduais, não é possível utilizar o modelo para analisar a relação entre condições de saúde e investimentos e ações de saneamento básico em outros municípios. Além disso, devemos considerar que o Brasil, por possuir enormes diferenças socioeconômicas entre as suas unidades da federação, pode discutir se um modelo que agregue todas as capitais, como o adotado aqui, é o mais adequado ou se análises regionalizadas representariam melhor as particularidades de cada parte do país.

Nesse sentido, futuros estudos podem ampliar a investigação sobre 0 impacto dos investimentos e ações de saneamento sobre a saúde para outros municípios, bem como realizar análises regionais ou de unidades da federação. Também é possivel investigar na literatura científica, evidências que apontem quais outras variáveis preditoras poderiam ser incorporadas ao modelo visando aumentar seu poder de explicação.

\section{REFERÊNCIAS}

BARCELLOS, Cristovam; MONTEIRO, Antônio MiguelVieira; CORVALÁN, Carlos; GURGEL, Helen C.; CARVALHO, Marília Sá; ARTAXO, Paulo; HACON, Sandra; RAGONI, Virginia. Mudanças climáticas e ambientais e as doenças infecciosas: cenários e incertezas para o Brasil. Revista Epidemiologia e Serviços de Saúde, Brasília, v. 18, n. 3, p. 285-304, jul/set, 2009.

BARBIERI, José Carlos; GIMENES, Régio Marcio Toesta; FAVERO, Marcela Bortotti; GIMENES, Fátima Maria P.; BORSATTO JÚNIOR, José Luiz. Investimentos em saneamento básico e taxas de mortalidade infantil nos municípios da região da AMUSEP. Revista de Ciências Empresariais UNIPAR, v. 16, n. 2, pp. 245-282, jul./dez. 2015.

BORJA, Patrícia Campos. Política pública de saneamento básico: uma análise da recente experiência brasileira. Revista Saúde e Sociedades, São Paulo, v. 23, n. 2, p. 432-447, 2014.

CRESWELL, John W. Projeto de pesquisa: métodos qualitativo, quantitativo e misto. Artmed, 2010.

NONATO, Domingos do Nascimento; DIAS, Daniella Maria dos Santos; RAIOL, Raimundo Wilson Gama.Saneamento e direito à cidade: Ponderações sobre abastecimento de água e esgotamento 
sanitário na cidade de Belém/PA. Revista de Direito da Cidade, Rio de Janeiro, v. 9, n. 4, p. 17841814, 2017.

FERREIRA, Patrícia da Silva Figueiredo; MOTTA, Patrícia Constantino; SOUZA, Tayane Crispim de; SILVA, Thiago Paulo da; OLIVEIRA, Juliana Ferreira de; SANTOS, Ana Silvia Pereira. Avaliação preliminar dos efeitos da ineficiência dos serviços de saneamento na saúde pública brasileira. Revista Internacional de Ciências, Rio de Janeiro, v. 6, n. 2, p. 214-229, jul/dez, 2016.

FIELD, Andy. Descobrindo a estatística usando o SPSS. 2. ed. Porto Alegre: Artmed, 2009.

FIRJAN - FEDERAÇÃO DAS INDÚSTRIAS DO ESTADO DO RIO DE JANEIRO. IFDM - Índice Firjan de Desenvolvimento Municipal - Ano Base 2016. Rio de Janeiro, jun., 2018.

HAIR JR, Joseph F.; ANDERSON, Rolph E.; TATHAM, Ronald L.; BLACK, William C. Análise multivariada de dados. 5. ed. Porto Alegre: Bookman, 2005.

HELLER, Léo. Relação entre saúde e saneamento na perspectiva do desenvolvimento. Ciência \& Saúde Coletiva, v. 3, n. 2, pp. 73-84, 1998.

HELLER, Léo; CASTRO, JoséEsteban. Política Pública de Saneamento: Apontamentos Teóricoconceituais. Engenharia Sanitária Ambiental, v. 12, n. 3, pp. 284-295, 2007.

IBGE - INSTITUTO BRASILEIRO DE GEOGRAFIA E ESTATÍSTICA. Divisão regional do Brasil. Disponivel em: https://www.ibge.gov.br/geociencias/organizacao-do-territorio/divisao-regional/15778divisoes-regionais-do-brasil.html?=\&t=o-que-e. Acesso em: 2 jul. 2019.

JUNG, Carlos Fernando. Metodologia para pesquisa \& desenvolvimento: aplicada a novas tecnologias, produtos e processos. Rio de Janeiro: Axcel Books do Brasil Editora, 2004.

LAHOZ, Rodrigo; DUARTE, Francisco. A Universalização do Serviço Público de Saneamento Básico e a Efetividade do Direito Fundamental à Saúde. Revista Direito e Justiça: Reflexões Sociojurídicas. v. 14, n. 23, pp. 117-130, fev. 2015.

LIMA, José Ronaldo Oliveira; SANTOS, Erica Luana Nunes dos; MEDEIROS, Jássio Pereira de. Saneamento e saúde pública: Análise das relações entre indicadores no Estado do Rio Grande do Norte. Revista Metropolitana de Sustentabilidade, São Paulo, v. 7, n. 2, p. 134-151, maio-ago, 2017.

MACIEL, Ana Beatriz Câmara; FELIPE, Jairo Alves; LIMA, Zuleide M. Carvalho. Os problemas de saneamento e seus impactos sobre a saúde pública do Município de Dona Inês/PB. Revista OKARA: Geografia em debate, João Pessoa, v. 9, n. 3, p. 524-541, 2015.

MADEIRA, Rodrigo Ferreira. O setor de saneamento básico no Brasil e as implicações do marco regulatório para a universalização do acesso. Revista BNDES, Rio de Janeiro, p. 123-154, jun. 2010. Disponivel em: https://web.bndes.gov.br/bib/jspui/bitstream/1408/4782/1/RB\%2033\%200\%20setor\%20de\%20saneame nto\%20b\%C3\%A1sico\%20no\%20Brasil_P.pdf. Acesso em: 9 jun. 2019.

MAROCO, João. Análise estatística com utilização do SPSS. 3. ed. Edições Sílabo, 2007. 
MELO, Francisca Jessica da Silva; SILVA FILHO, José Adalberto da; ANDRADE, Silvana Nóbrega de; VIEIRA, Z. Caetano. Análise do saneamento básico e saúde pública na Cidade de Pombal, Paraíba. Revista Verde de Agroecologia e Desenvolvimento Sustentável, Pombal, v. 12, n. 1, p. 74-78, 2017.

MENDES, Thiago Mendes; BARCELLOS, Christovam. A dimensão territorial do esgotamento sanitário: o caso do Recreio dos Bandeirantes, Rio de Janeiro, Brasil. Revista Ciência e Saúde Coletiva, v. 23, n. 2, p. 647-658, 2018.

MORAES, Luiz Roberto Santos; BORJA, Patrícia Campos. Revisitando o conceito de saneamento básico no Brasil e em Portugal. Revista do Instituto Politécnico da Bahia, v. 20, n. 7, p. 5-11, 2014.

MOTA, João José Pessoa; SOUSA, Carlos Di Stefano Silva; SILVA, Alessandro Costa da. Saneamento básico e seu reflexo nas condições socioambientais da Zona Rural do Baixo Munim (Maranhão).

Revista Online Caminhos da Geografia, Uberlândia, v. 16, n. 54, p. 140-160, 2015.

MOURA, Diogo Felipe Santos de; TROLEIS, Adriano Lima; SILVA, Bruno Lopes da. Saneamento básico e saúde púbica: uma análise dos níveis de Nitrato da água consumida pela população do bairro Pajuçara - Natal/RN. Revista de Geociências do Nordeste, v. 2, número especial, 2016.

ROSA, Fabrícia Silva da; DÍAZ-BECERRA, Oscar A.; LUNKES, Rogério João. Saneamento básico: Análise da relação entre gastos públicos e atendimento à população em cidades brasileiras e peruanas. Revista Científica General José Maria Córdova, Bogotá, Colombia, v. 14, n. 18, p. 195-213, jul-dez, 2016.

SAIANI, Carlos César Santejo; TONETO JR., Rudinei. Evolução do acesso a serviços de saneamento básico no Brasil (1970 a 2004). Revista Economia e Sociedade, Campinas, v. 19, n. 1 (38), p. 79-106, 2010.

SILVA, Suzana de Araújo; GAMA, José Aparecido da Silva; CALLADO, Nélia Henriques; SOUZA, Vladimir Caramori Borges de. Saneamento básico e saúde pública na Bacia Hidrográfica do Riacho Reginaldo em Maceió, Alagoas. Revista Engenharia Sanitária e Ambiental, Rio de Janeiro, v. 22, n. 4, p. 699-709, jul/ago, 2017.

SOARES, Sérgio R. A.; BERNARDES, Ricardo S.; CORDEIRO NETTO, Oscar de M. Relações entre saneamento, saúde pública e meio ambiente: elementos para formulação de um modelo de planejamento em saneamento. Caderno Saúde Pública, Rio de Janeiro, v. 18, n. 6, p. 1713-1724, nov/dez, 2002.

TEIXEIRA, Júlio C.; GUILHERMINO, Renata Lopes. Análise da associação entre saneamento e saúde nos estados brasileiros, empregando dados secundários do banco de dados indicadores e dados básicos para a saúde 2003- IDB 2003. Engenharia Sanitária Ambiental, v.11, n.3, pp.277-282, 2006.

TEIXEIRA, Júlio C.; OLIVEIRA, Guilherme Soares de; VIALI, Amanda de Mello; MUNIZ, Samuel Soares. Study of the impact of deficiencies of sanitation on public health in Brazil from 2001 to 2009. Engenharia Sanitária Ambiental, v. 19, n. 1, pp. 87-96, 2014.

TOIGO, Leandro Augusto; WRUBEL, Franciele; HEIN, Nelson. Influências contingenciais no custo de produção de suínos em ciclo completo no Estado de Santa Catarina. Custos e @gronegócios online. v. 10, n. 4, p. 198-221, 2014. 